UDC $613,614,616.9$

DOI: $10.21668 /$ health.risk/2019.3.14.eng

\title{
MULTISEPARATION OF ANTHOCYANINS AND ANTHOCYANIDINS BY HIGH PERFORMANCE LIQUID CHROMATOGRAPHY COMBINED WITH RESPONSE SURFACE METHODOLOGY
}

\author{
Vu Thi Trang ${ }^{1,2}$, Le Hoang Duc ${ }^{2}$, Nguyen Hoai Thu ${ }^{2}$, Le Thi Hong Hao ${ }^{1,2}$, \\ Nguyen Xuan Trung ${ }^{2}$ \\ ${ }^{1}$ National Institute for Food Control, 65 Pham ThanDuat, Hanoi, Vietnam \\ ${ }^{2}$ VNU University of Science, Vietnam National University-Hanoi, 19 Le Thanh Tong, Hanoi, Vietnam
}

\begin{abstract}
A method has been developed for the separation of anthocyanins and anthocyanidins with high performance liquid chromatography (HPLC). Experimental designs were applied for multivariate optimization of the HPLC experimental conditions with using response surface methodology (RSM). Three independent factors, namely formic acid concentration, initial ratio of acetonitrile in gradient program and flow rate, were used to design mathematical models. The responses were resolutions of four peak couples which were the most difficult to separate including: pelargonidin-3-glucoside and delphinidin, delphinidin and peonidin-3-glucoside, peonidin-3-glucoside and malvidin-3-glucoside, peonidin and malvidin. Variance analysis proved a chosen model was highly fit and the RSM method yielded good results in improving separation of anthocyanins and anthocyanidins. The optimized HPLC parameters were as follows: C18 column $(250 \mathrm{~mm} \times 4.6 \mathrm{~mm} \times 5 \mu \mathrm{m})$, mobile phase is FA $10 \%$ and ACN used as a gradient; flow rate $0.8 \mathrm{~mL}$.min-1. Using these optimum conditions, separation of compounds with good resolutions and a run time of less than $30 \mathrm{~min}$ were archived. The results for method validation satisfied the requirement of AOAC, linearity range from $0.2-10 \mathrm{ppm}$ with $R^{2} \geq 0.9955, L O D$ from $0.05-0.1 \mathrm{mg} / \mathrm{kg}, \mathrm{RSD}$ from $4.79-6.45 \%$ and the recovery is from $85.4-109.6 \%$. The method was applied to determone anthocyanins and anthocyanidins in some fruits and vegetables samples with the content of anthocyanidins being from $5.74-218.27 \mathrm{mg} / 100 \mathrm{~g}$. Anthocyanins primarily concentrate in peel of fruits and vegetables, black bean peel contains most anthocyanins.
\end{abstract}

Key words: anthocyanin, anthocyanidin, response surface methodology (RSM), HPLC, antioxidant, chromatography.

Introduction. The anthocyanins are the largest and most important group of watersoluble pigments which are responsible for the blue, purple, red and orange colors of numerous fruits and vegetables. Anthocyanins occur naturally as glycosides of their respective aglycone (anthocyanidin) nucleus with the sugar moiety. The differences between anthocyanins are related to the number of hydroxyl groups, the nature and number of sugars attached to the molecule, the position of these sugars, and the nature and number of aliphatic or aromatic acids attached to the sugars [12]. It is reported that more than 600 different anthocyanins have been found in plant sources [20]. Most of them were formed from the six most common anthocyanidins including cyanidin (Cya), delphinidin
(Del), petunidin (Petu), pelargonidin (Pelar), malvidin (Mal) and peonidin (Peo) [3]. In nature, anthocyanins rarely exist in the free state (unglycosylated) because the lack of electrons in the cationic flavylium makes it very reactive and therefore the molecule is very unstable [13]. Sugar attachment is thought to be of importance for pigment stability. They occur in flower mainly as 3-monoglucosides, 3,5-diglucosides or 3,7-diglycosides [13].

Anthocyanins possess potent antioxidant capacities and health-promoting properties. With such advantages and popularity, anthocyanins and anthocyanidins compounds are replacing artificial food colorants.

The daily intake of anthocyanins in the U.S. diet is estimated to be between 180 and

(C) Vu Thi Trang, Le Hoang Duc, Nguyen Hoai Thu, Le Thi Hong Hao, Nguyen Xuan Trung, 2019

Vu Thi Trang - PhD., candidate, head of Laboratory of Food Quality and Food Additives (e-mail: trang2103@gmail.com; tel.: +84916149106).

Le Hoang Duc - Student (e-mail: duclhd2909@gmail.com; tel.: +84973552366).

Nguyen Hoai Thu - Student (e-mail: nguyenhoaithu2596@gmail.com; tel.: +84359673139).

Le Thi Hong Hao - PhD., general director (e-mail: lethihonghao@yahoo.com; tel.: +84904248167).

Nguyen Xuan Trung - PhD., lecturer (e-mail: trungnx2004@,gmail.com; tel.: +84904303513). 
$215 \mathrm{mg}$, whereas the intake of other dietary flavonoids such as genistein, quercetin or andapigenin is only 20-25 mg.day ${ }^{-1}$ [15]. Anthocyanins are postulated to act as antioxidants by donating hydrogen atoms to form highly reactive free radicals [5].

There are numbers of methods applied to determine anthocyanins or anthocyanidins such as UV-Vis [2], HPLC [19, 11, 9], LC-MS $[7,8,23,1]$ or $\mathrm{CE}[17,6]$. However, the published studies usually applied these techniques to determine the total content of anthocyanins or anthocyanidins. It has been reported that a simultaneous determination of anthocyanin glucosides and anthocyanidin aglycones is challenged due to different polarities between the anthocyanin glucoside group and the anthocyanidin aglycone group [18]. Therefore, this study focused on the multi-separation of twelve anthocyanins and anthocyanidins by HPLC combined with response surface methodology.

Experimental. Materials and reagents. Anthocyanidins standards including Delphinidin chloride, cyanidin chloride, pelargonidin chloride, peonidin chloride and malvidin chloride were provided by Sigma Aldrich. Petunidin chloride was provided by Chromadex. Anthocyanins standards: Pelargonidin - 3 - glucoside (Pelar-3-G), cyaniding - 3 - glucoside (Cya-3-G), peonidin 3 - glucoside (Peo-3-G) and malvidin - 3 glucoside (Mal-3-G) were purchased from Sigma - Aldrich. Delphinidin - 3 - glucoside (Del-3-G) and Petunidin - 3 - Glucoside (Petu-3-G) are from Chromadex. All other reagents and chemicals were of analytical or HPLC grade from Merck.

Several fruits and vegetables (red apple, purple potato, black grape, black bean, etc.) were randomly collected from the markets and supermarkets in Hanoi, Vietnam, and analyzed using the optimized method.

Apparatus and procedures. All experiments were conducted on a Shimadzu LC 20AD series liquid chromatography equipped with a vacuum degasser, binary pump, autosampler, column oven and photodiode array detector. Instrument control and data acquisi- tion and analysis were performed through LC Solution software.

Separation was achieved on a Sun Fire $\mathrm{C}_{18}$ column $250 \mathrm{~mm} \times 4.6 \mathrm{~mm}, 5 \mu \mathrm{m}$ with a guard column. The injection volume was $20 \mu \mathrm{L}$ with PDA detection at $520 \mathrm{~nm}$. The elution solvents were formic acid in water and acetonitrile at specific compositions.

A homogenized sample was weighted into a heat-resistant flask. $30 \mathrm{~mL}$ of methanol was then added to the flask. The solution was extracted at $100^{\circ} \mathrm{C}$ during $30 \mathrm{~min}$. After cooling, the extract was diluted and filtered by using $0.45 \mu \mathrm{m}$ membrane. Obtained solution was finally analyzed on HPLC system with PDA detector for anthocyanin.

For anthocyanidin detection, the anthocyanin extract was added with concentrated hydrochloric acid to reach hydrochloric acid concentration of $2.4 \mathrm{M}$ and heated in water bath at $90^{\circ} \mathrm{C}$ in $60 \mathrm{~min}$. After cooling to the room temperature, the solution was diluted by adding methanol, filtered and analyzed on HPLC system with PDA detector for anthocyanidin.

Data analysis. The data analysis was performed using Design-Expert Version 10.0.7 statistical software (Stat-Ease Inc., Minneapolis, MN, USA). Factor significance was calculated using such a statistical tool as analysis of variance (ANOVA) that was estimated and run up to the first order interaction terms. For all the calculation, it was assumed that higher order interaction terms did not contribute significantly to the behavior of the selected statistical model, since the chances of these interactions happening are low.

Method validation. The optimized method was validated with the following parameters: linearity and range, precision, accuracy, and limit of detection and quantitation. The results were verified according to AOAC strict validation guidelines.

Results and discussions. Selection of HPLC analytical conditions. Due to the polarity of anthocyanins and anthocyanidins the nonpolar column $\mathrm{C}_{18}$ was chosen as the separate column. It was reported that the anthocyanidins and anthocyanins were eluted by ace- 
tonitrile (ACN) and acid, such as: formic acid (FA) concentration of $0.05-10 \%$, trifluoroacetic acid (TFA) concentration of $0.05-0.1 \%$, phosphoric acid $0.3-4 \%$, and hydrochloric acid $5 \mathrm{mM}[10,14,16,4,21,22]$. In particular, FA and TFA acid were commonly used because organic acids increase the signal and should be added to water as eluent. The use of inorganic acids such as hydrochloric acid or phosphoric acid at high concentrations could affect the chromatographic column performance. Therefore, in this study FA and TFA were selected to investigate the separation of anthocyanidins and anthocyanins.

Preliminary experiments showed that using TFA $0.1 \%$ produced sharp, symmetrical but overlapped peaks, while FA $0.1 \%$ completely separated the peaks. On the other hand, as FA is a weaker acid than TFA, it was possible to increase FA concentration to obtain higher sensitivity (sharper peak). Therefore, FA was chosen for optimizing the separation.

Due to the different polarities, anthocyanidins and anthocynins interact differently with stationary phases. When eluting in an isocratic mode they could be completely separated from each other, but there is still a possibility that the analysis time lasts approximately 50 to 60 minutes and the signal could be significantly low. For this reason, it is necessary to change the ratio of the mobile phase composition to the gradient program.

In addition, the flow rate could strongly affect the elution capacity, the amount of solvent consumed, and the analysis time. For the $\mathrm{C}_{18}$ column $(250 \mathrm{~mm} \times 4.6 \mathrm{~mm} \times 5 \mu \mathrm{m})$ the flow rate was selected in the range of $0.6-1.0 \mathrm{~mL} / \mathrm{min}^{-1}$

In this part, investigates were archived to find out a condition in which the resolution of 4 couples of peaks (Pelar-3-G and Del, Del and Peo-3-G, Peo-3-G and Mal-3G, Peo and Mal) was larger than 2 for a good determination.

Optimization of analytical factors by RSM. RSM is a collection of mathematical and statistical techniques for empirical model building. By careful design of experiments, the objective is to optimize a response (output variable) which is influenced by several independent variables (input variables). An experiment is a series of tests, called runs, in which changes are made in the input variables in order to identify the reasons for changes in the output response.

In order to determine simultaneously twelve anthocyanins and anthocyanidins, a proper separation between them was absolutely important. Hence, it was necessary to find a condition in which the peaks were separated completely, it means that the resolutions of peaks were large enough (normally larger than 2). Aquadratic central composite designwas used with 3 independent factors, including the concentration of FA in mobile phase $\left(\mathrm{X}_{1}\right)$, the initial ratio of $A C N$ in the gradient $\left(\mathrm{X}_{2}\right)$, and the flow rate $\left(\mathrm{X}_{3}\right)$. The responses were the resolutions of peaks that are usually small. According to our preliminary results, four responses were selected as follows: the resolution between Pelar-3-G and Del $\left(\mathrm{Y}_{1}\right)$, Del and Peo-3-G $\left(\mathrm{Y}_{2}\right)$, Peo-3-G and Mal-3-G $\left(\mathrm{Y}_{3}\right)$, and Peo and Mal $\left(\mathrm{Y}_{4}\right)$.

The number of experiments performed on the quadratic central composite matrix was calculated by the following formula:

$$
N=N_{\mathrm{o}}+N^{*}+N_{\mathrm{c}},
$$

where:

$\mathrm{N} *$ is a number of experiments at point *, $\mathrm{N}^{*}=2 \mathrm{n}$

$\mathrm{N}_{\mathrm{c}}$ is a number of experiments at center, $\mathrm{N}_{0}>1$

$\mathrm{N}_{\mathrm{o}}$ is a number of experiments at origin, $\mathrm{N}_{o}=2^{\mathrm{n}-\mathrm{q}}$ ( $\mathrm{n}$ is a number of factors, $\mathrm{q}$ is a number of reduced factors)

For $\mathrm{n}=3, \mathrm{q}=0$, we have the following: $\mathrm{N}^{*}=6 ; \mathrm{N}_{\mathrm{o}}=8 ; \mathrm{N}_{\mathrm{c}}=6$

Thus, the total number of experiments proceeded $\mathrm{N}=20$ with 6 repeated experiments at the center and the distance from the origin to the point $*$ is $d=2^{(n-q) / 4}=1.682$.

By using the Design Expert software, the quadratic response with three factors was evaluated and the results are given in Table 1.

Table 1

The base levels of 3 factors with their codes

\begin{tabular}{|l|c|c|c|}
\hline \multirow{2}{*}{ Factors } & \multicolumn{3}{|c|}{ Level } \\
\cline { 2 - 4 } & -1 & 0 & +1 \\
\hline$X_{1}$ (concentration of FA, \% v/v) & 5 & 10 & 15 \\
\hline$X_{2}$ (initial ratio of ACN, \%) & 1 & 6,5 & 12 \\
\hline$X_{3}$ (flow rate, mL.min-1) & 0,6 & 0,8 & 1,0 \\
\hline
\end{tabular}


The design matrix as well as the corresponding resolution values are given in Table 2 . All experiments were performed in randomized order to minimize the effects of uncontrolled variables that may introduce a bias in the measurements.

By postulating a fitted full quadratic model described in Eq. (1), the mathematical models were obtained for each response $\left(\mathrm{Y}_{\mathrm{i}}\right)$ in terms of coded factors after fitting Eq. (1) by the least square regression, see Table 3.

$$
Y_{i}=b_{0}+\sum_{i=1}^{k} b_{i} x_{i}+\sum_{1 \leq i \leq j}^{k} b_{i j} x_{i} x_{j}+\sum_{i=1}^{k} b_{i i} x_{i}^{2}
$$

Where $\mathrm{k}$ is the number of factors (variables); $\mathrm{b}_{0}$ is the intercept parameter; and $b_{i}, b_{i j}$ and $b_{i i}$ are the regression parameters for linear, interaction, and quadratic effects of each factor $\mathrm{x}_{\mathrm{i}}$, respectively.

Table 2

Experimental design matrix of coded variables and studied responses

\begin{tabular}{|c|c|c|c|c|c|c|c|c|}
\hline \multirow{2}{*}{$\begin{array}{c}\text { Std. } \\
\text { Ord. }\end{array}$} & Run & \multicolumn{3}{|c|}{ Coded variable } & \multicolumn{4}{c|}{ Response } \\
\cline { 3 - 8 } & Ord. & $X_{1}$ & $X_{2}$ & $X_{3}$ & $Y_{1}$ & $Y_{2}$ & $Y_{3}$ & $Y_{4}$ \\
\hline 19 & 1 & 10 & 6,5 & 0,8 & 2,641 & 3,483 & 2,344 & 2,696 \\
\hline 3 & 2 & 5,0 & 12 & 0,6 & 5,044 & 0,468 & 4,525 & 2,394 \\
\hline 15 & 3 & 10 & 6,5 & 0,8 & 2,637 & 3,609 & 2,381 & 2,717 \\
\hline 4 & 4 & 15 & 12 & 0,6 & 1,838 & 1,566 & 3,206 & 3,415 \\
\hline 9 & 5 & 1,6 & 6,5 & 0,8 & 1,589 & 0,729 & 3,227 & 1,601 \\
\hline 1 & 6 & 5,0 & 1,0 & 0,6 & 1,229 & 0,000 & 0,548 & 0,140 \\
\hline 8 & 7 & 15 & 12 & 1,0 & 2,271 & 2,272 & 2,988 & 3,122 \\
\hline 10 & 8 & 18,4 & 6,5 & 0,8 & 0,685 & 3,040 & 3,345 & 3,549 \\
\hline 7 & 9 & 5,0 & 12 & 1,0 & 4,479 & 0,611 & 3,522 & 2,579 \\
\hline 20 & 10 & 10 & 6,5 & 0,8 & 2,641 & 3,483 & 2,344 & 2,696 \\
\hline 6 & 11 & 15 & 1,0 & 1,0 & 1,527 & 3,010 & 2,593 & 3,438 \\
\hline 11 & 12 & 10 & 0,0 & 0,8 & 1,250 & 0,832 & 0,717 & 2,495 \\
\hline 14 & 13 & 10 & 6,5 & 1,1 & 2,663 & 3,500 & 2,143 & 2,405 \\
\hline 12 & 14 & 10 & 15,7 & 0,8 & 4,560 & 0,000 & 3,878 & 3,639 \\
\hline 13 & 15 & 10 & 6,5 & 0,5 & 2,669 & 1,271 & 1,817 & 0,943 \\
\hline 5 & 16 & 5,0 & 1,0 & 1,0 & 0,000 & 1,673 & 0,997 & 1,932 \\
\hline 16 & 17 & 10 & 6,5 & 0,8 & 2,630 & 3,438 & 2,325 & 2,717 \\
\hline 18 & 18 & 10 & 6,5 & 0,8 & 2,639 & 3,430 & 2,326 & 2,695 \\
\hline 2 & 19 & 15 & 1,0 & 0,6 & 1,436 & 0,877 & 1,382 & 2,427 \\
\hline 17 & 20 & 10 & 6,5 & 0,8 & 2,625 & 3,462 & 2,338 & 2,713 \\
\hline
\end{tabular}

Table 3

Refined regression equations and statistical parameters for studied responses from the experimental design

\begin{tabular}{|c|c|c|c|c|c|}
\hline Response & Regression equations $^{\mathrm{a}}$ & $\begin{array}{c}\mathrm{C} . \mathrm{V} . \\
(\%)\end{array}$ & $\begin{array}{c}\text { Adjusted } \\
\mathrm{R}^{2}\end{array}$ & $\begin{array}{c}\text { Predicted } \\
\mathrm{R}^{2}\end{array}$ & $\begin{array}{c}\text { Adequate } \\
\text { precision }\end{array}$ \\
\hline$Y_{1}^{\mathrm{c}}$ & $\begin{array}{c}Y_{1}=2,72-0,38 X_{1}+1,10 X_{2}-0,094 X_{3}- \\
-0,89 X_{1} X_{2}+0,29 X_{1} X_{3}-0,54 X_{1}^{2}\end{array}$ & 9,22 & 0,9707 & 0,9286 & 37,040 \\
\hline$Y_{2}$ & $\begin{array}{c}Y_{2}=3,49+0,65 X_{1}-0,15 X_{2}+0,62 X_{3}+0,13 X_{1} X_{3}- \\
-0,37 X_{2} X_{3}-0,59 X_{1}{ }^{2}-1,11 X_{2}^{2}-0,42 X_{3}^{2}\end{array}$ & 7,35 & 0,9878 & 0,9628 & 34,651 \\
\hline$Y_{3}^{\mathrm{c}}$ & $\begin{array}{c}Y_{3}=2,32+0,057 X_{1}+1,03 X_{2}+0,072 X_{3}-0,54 X_{1} X_{2}+ \\
+0,19 X_{1} X_{3}-0,36 X_{2} X_{3}+0,32 X_{1}{ }^{2}-0,14 X_{3}^{2}\end{array}$ & 4,12 & 0,9904 & 0,9705 & 57,652 \\
\hline$Y_{4}$ & $\begin{array}{c}Y_{4}=2,67+0,63 X_{1}+0,40 X_{2}+0,38 X_{3}-0,28 X_{1} X_{2}- \\
-0,16 X_{1} X_{3}-0,36 X_{2} X_{3}+0,13 X_{2}{ }^{2}-0,36 X_{3}^{2}\end{array}$ & 4,44 & 0,9830 & 0,9433 & 46,720 \\
\hline
\end{tabular}

${ }^{\text {a }}$ Obtained by applying backward elimination tool to remove nonsignificant $(\mathrm{P}>0.05)$ terms from the full models

${ }^{\mathrm{b}}$ Coefficient of variation

${ }^{\mathrm{c}}$ The nonsignificant terms $\mathrm{X}_{3}\left(\right.$ in $\left.\mathrm{Y}_{1}\right), \mathrm{X}_{1}$ (in $\mathrm{Y}_{3}$ ) were included in the equation to maintain model hierarchy. 
As there are potential problems associated with the normality assumption, unequal error variance by treatment or block, and block treatment interaction, the adequacy of the assumed model needs to be examined. In this study, adequacy checking of ultimate refined models was carried out using the adequate precision statistic tool and normal probability plots of the residuals. "Adequate Precision" measures the signal to noise ratios. A ratio greater than 4 is desirable. The ratios of $37.040,34.651,57.652$, and 46.720 indicate adequate signals. This model can be used to navigate the design space. Besides that, a fitted model is usually evaluated with a coefficient of determination, $\mathrm{R}^{2}$. A concern with this statistic value is that it always increases when terms are added to the model, even if the terms are not significant. Therefore, it is usually smaller for the refined model than the corresponding full model. To overcome the disadvantages associated with the use of $\mathrm{R}^{2}$, adjusted coefficients, adjusted $\mathrm{R}^{2}$, are used. Consequently, to get a simpler but more accurate model, the nonsignificant terms $(P>0.05)$ were removed from the models. Although the main effect $\mathrm{X}_{3}$ was not a significant term in models corresponding to $Y_{1}$, and $X_{1}$ was not a significant term in models corresponding to $\mathrm{Y}_{3}$ to comply with the model hierarchy, it was included in the resulting equations. In addition, the values fell within the reasonable agreement with predicted $\mathrm{R}^{2}$ and within the acceptable limits of $\mathrm{R}^{2} \geq 0.80$, indicating that the data showed good fit with the second-order polynomial equations. The values of model $\mathrm{F}$ implied that all four models were significant. There was only a $0.01 \%$ chance that an F-value this large could occur due to noise. The values obtained for the coefficient of variation (C.V.) percent, which measures the reproducibility of a model, were less than $10 \%$ (from $4.12 \%$ to $9.22 \%$ ).

As we can see in Table 3 and Figure 1, the factors had very different effects on responses. Concentration of formic acid and flow rate made positive effects on responses $\mathrm{Y}_{2}, \mathrm{Y}_{3}, \mathrm{Y}_{4}$ but negative effects on response $Y_{1}$. Concentration of acetonitrile made positive effects on responses $Y_{1}, Y_{3}, Y_{4}$ but negative effects on response $Y_{2}$. For $Y_{1}$ resolution (pelar-3-G and Del), $\mathrm{Y}_{3}$ resolution (Peo-3-G and Mal-3-G), the term $X_{2}$ had the most significant effect on separation. These resolutions were increased by $\mathrm{X}_{2}$. Interaction of formic acid and acetonitrile increased the resolutions, while interaction of acetonitrile and flow rate decreased them. Almost square terms had negative effects. The flow rate had little effect on the responses than the other two, possibly due to the limited operating range.

Figure 1B showed the effect of formic concentration and flow rate in the resolution of Peo and Mal by keeping acetonitrile ratio at $6.5 \%$. As we can see, increasing both formic acid concentration and flow rate led to a gradual increase in the resolution. This can be explained by anthocyanins and anthocyanidins being stable at $\mathrm{pH}<3$. A higher acid concentration and lower $\mathrm{pH}$ level could raise the percentage of flavylium cation and increase the peak area and resolution. However, high organic acids could increase the polarity of the mobile phase, resulting in weaker power of elution and thus a longer retention time. Subsequent reduction in the retention time could be attributed to a competitive interaction between formic acid molecules and the polar groups of anthocyanins for the silanol group on the alkyl silica surface when excessive organic acid was added.

When a simple response is being analyzed, model analysis indicates areas in the design region where the process is likely to give desirable results. Many response surface problems involve the analysis of several responses. Meanwhile, simultaneous consideration of multiple responses involves building an appropriate response surface model for each response and finding a set of operating conditions that in some senses optimizes 


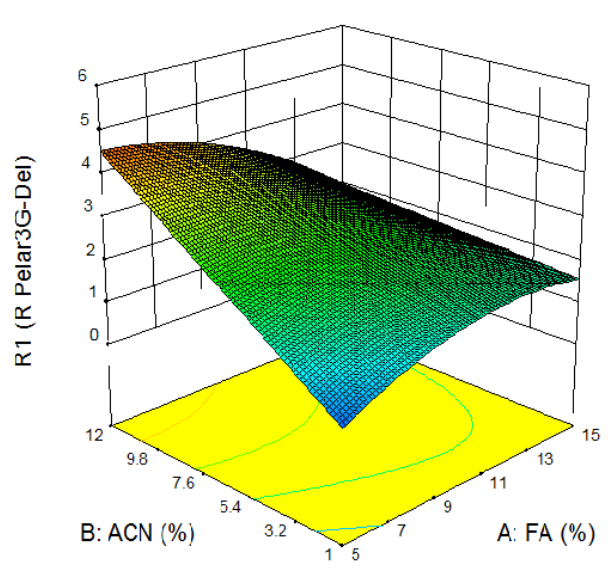

(A) The resolution of Pelar-3-G and Del as a function of acetonitrile ratio and formic concentration

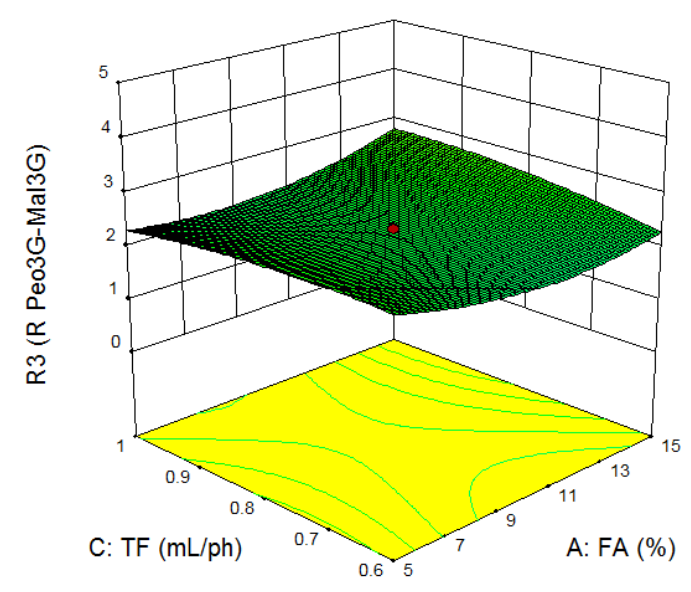

(C) The resolution of Peo-3-G and Mal-3-G as a function of flow rate and formic concentration

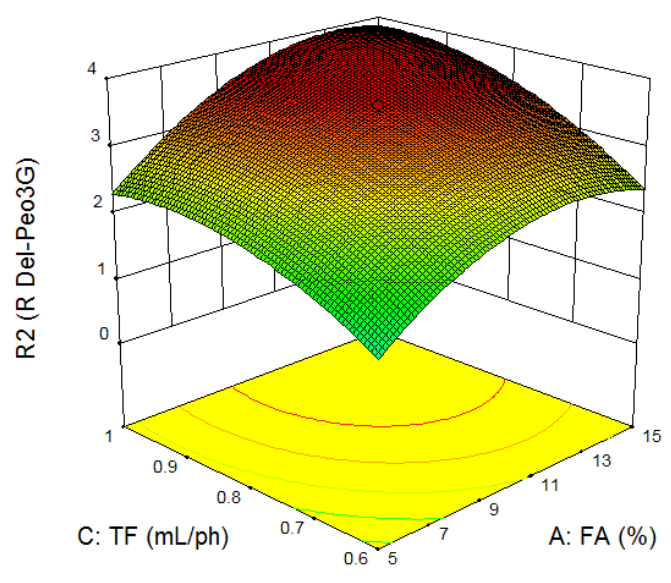

(B) The resolution Del and Peo-3-G as a function of flow rate and formic concentration

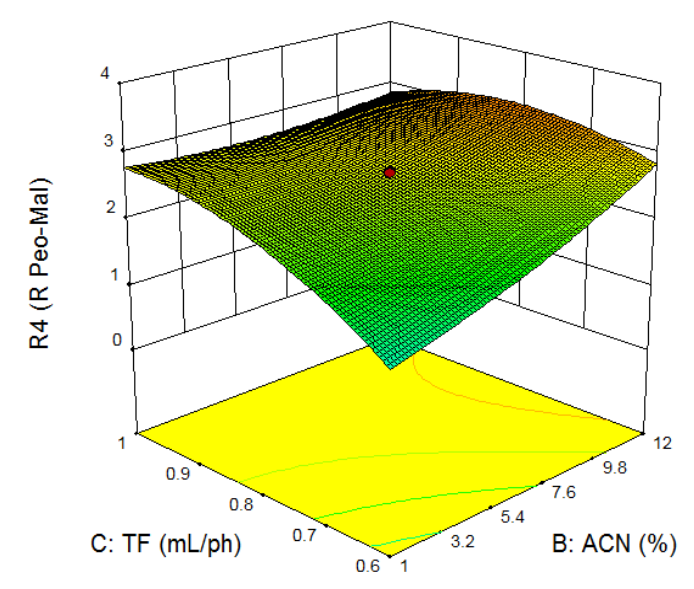

(D) The resolution of Peo and Mal as a function of flow rate and acetonitrile ratio

Figure 1. Response surface plots representing the responses

all responses or at least keeps them within a desired range. Settings for multi-criteria optimization of the individual factors and responses were showed in Table 4.

The goals were combined employing this strategy into an overall desirability function and the global desirability for the optimal solution was determined to be 0.748 . Following the conditions and restrictions discussed, the optimal calculated parameters were obtained as: formic acid concentration of $10 \%$, acetonitrile ratio of $10 \%$ and flow rate of 0.80 mL.min-1.
Table 4

Settings for multi-criteria optimization of the individual factors and responses

\begin{tabular}{|c|c|c|c|c|}
\hline $\begin{array}{c}\text { Factor/ } \\
\text { Response }\end{array}$ & Goal & $\begin{array}{c}\text { Lower } \\
\text { limit }\end{array}$ & $\begin{array}{c}\text { Upper } \\
\text { limit }\end{array}$ & Importance \\
\hline$X_{1}$ & In range & 5 & 10 & 3 \\
\hline$X_{2}$ & Inrange & 1 & 12 & 3 \\
\hline$X_{3}$ & Target $=1$ & 0,6 & 1 & 3 \\
\hline$Y_{1}$ & Maximum & 0 & 5,044 & 2 \\
\hline$Y_{2}$ & Maximum & 0 & 3,609 & 5 \\
\hline$Y_{3}$ & Maximum & 0 & 4,525 & 5 \\
\hline$Y_{4}$ & Maximum & 0 & 3,639 & 4 \\
\hline
\end{tabular}




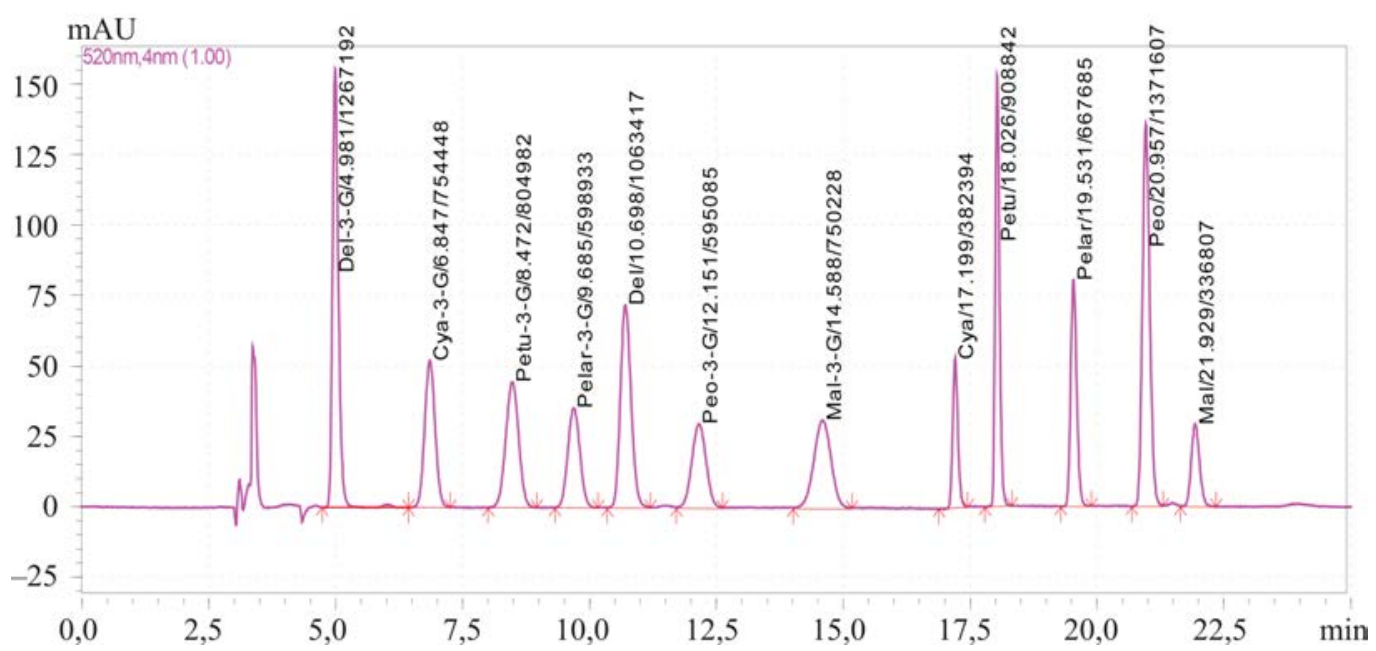

Figure 2.Chromatogram of twelve anthocyanin and anthocyanidin at optimized conditions. The elution order was as follow: (1) Del-3-G, (2) Cya-3-G, (3) Petu-3-G, (4) Pelar-3-G,

(5) Del, (6) Peo-3-G, (7) Mal-3-G, (8) Cya, (9) Petu, (10) Pelar, (11) Peo, (12) Mal.

The optimized HPLC parameters: C18 Sun Fire column by Waters $(250 \mathrm{~mm} \times 4.6 \mathrm{~mm} \times$ $5 \mu \mathrm{m})$, mobile phase was FA $10 \%$ and $\mathrm{ACN}$ using a gradient: starting at $10 \% \mathrm{ACN}$, linearity increasing from $10-12 \% \mathrm{ACN}$ in $0-10$ minutes, constant at $19 \%$ ACN from $10.01-$ 21 minutes, constant at $10 \% \mathrm{ACN}$ in $21.01-$ 25 minutes for the next analysis; flow rate: 0.8 mL.min-1. Fig. 2 was the chromatogram of multi-separation anthocyanins and anthocyanidins with good resolutions.

Method validation. Calibration curve of anthocyanins and anthocyanidins was established in the concentration range of $0.2-10$ $\mathrm{mg} / \mathrm{L}$ with the correlation coefficient $>0.9955$. Limit of detection was determined by gradually spiking small amounts of standard solution into blank samples until getting the signal over noise equal to 3 . The results showed that HPLC-PDA method provided a good sensitivity for the determination of anthocyanins and anthocyanidins with LOD of $0.05-0.1 \mathrm{mg} / \mathrm{kg}$. Precision was assessed by six replicates in some sample matrixes. RSD was from $4.79-6.45 \%$. The recovery was determined by spiking standards into plum, sweet potato samples with three different levels. Assays were performed in three replicates at each added concentration. The recoveries between
$85.4 \%$ and $109.6 \%$ have been obtained. The method satisfied the requirement of recovery for corresponding analyte concentration according to AOAC.

Analysis of the marketed products. The samples were collected randomly from the markets and supermarkets in Hanoi. After homogenizing, the samples were analyzed by the procedure as mentioned above.

After the extraction, almost anthocyanins were transformed to anthocyanidins. The anthocyanins content before the hydrolysis and anthocyanidins content after the hydrolysis of some fruits were given in Tables 5 and Figure 3.

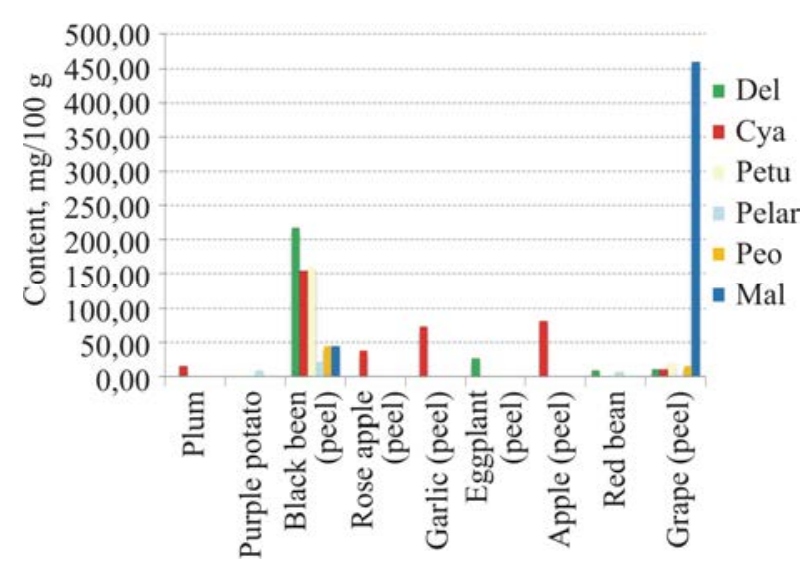

Figure 3. The anthocyanidins contents in marketed products 
Table 5 thocyanidins and also had the highest total con-

Anthocyanins content in the marketed products

\begin{tabular}{|l|c|c|c|c|}
\hline \multicolumn{1}{|c|}{ Samples } & \multicolumn{4}{|c|}{ Content (mg/100g) } \\
\hline Plum & Cya-3-G & Pelar-3-G & Peo-3-G & Mal-3-G \\
\hline $\begin{array}{l}\text { Purple } \\
\text { potato }\end{array}$ & 1.57 & - & - & - \\
\hline $\begin{array}{l}\text { Black bean } \\
\text { (peel) }\end{array}$ & - & 1.05 & - & - \\
\hline $\begin{array}{l}\text { Rose apple } \\
\text { (peel) }\end{array}$ & 18.67 & - & 8.28 & 19.30 \\
\hline Garlic (peel) & 11.23 & - & - & - \\
\hline $\begin{array}{l}\text { Eggplant } \\
\text { (peel) }\end{array}$ & 8.4 & - & - & - \\
\hline Apple (peel) & - & - & - & - \\
\hline Red bean & 27.17 & - & - & - \\
\hline Grape (peel) & 1.76 & 7.40 & - & - \\
\hline
\end{tabular}

Accordingly, the anthocyanidins content was much higher than the anthocyanins-3glucoside content, so, there were other types of anthocyanins in the samples excluding anthocyanins-3-glucoside. Among them, cyanidin-3glucoside was the most appeared anthocyanin in fruits and vegetables, other anthocyanins were found in a less extent. In addition, cyanidin was the most common anthocyanidin, which occurs in almost sample, following by delphinidin.

Black bean peel had the biggest diversity of anthocyanidins with all 6-most-common an- tent, reaching more than $600 \mathrm{mg}$ anthocyanidins in $100 \mathrm{~g}$ sample. Grape peel also contained a high total content of anthocyanidins, with the majority was malvidin, which reaches more than $450 \mathrm{mg} / 100 \mathrm{~g}$. Plum, rose apple peel, garlic peel and apple peel contained only cyanidin. Purple potato and eggplant contained only pelargonidin and delphinidin, respectively.

Conclusions. The main contribution of this study was to use experimental design to find optimal HPLC conditions for simultaneous separation twelve anthocyanins and anthocyanidins. The mathematical models developed for relating resolutions to the composition of the mobile phase proved to be an efficient strategy for optimization of the chromatographic method. A significant good fit with the models was found between predicted and observed data, which means the method was suitable for the analysis of the compounds. This method allows simultaneous determination of anthocyanins and anthocyanidins in fruits and vegetables samples in a short time, with a high selectivity and sensitivity. The procedure is simple, and the cost is also reasonable.

Funding. The research was not granted any sponsor support.

Conflict of interests. The authors state there is no any conflict of interests.

\section{References}

1. Bordonaba J.G., Crespo P., Terry L.A. A new acetonitrile-free mobile phase for HPLC-DAD determination of individual anthocyanins in blackcurrant and strawberry fruits: A comparison and validation study. Food Chemistry, 2011, no. 129, pp. 1265-1273. DOI: 10.1016/j.foodchem.2010.09.114

2. Deineka V.I., Deineka L.A., Saenko I.I. Regularities of Anthocyanins Retention in RP HPLC for «Water-Acetonitrile-Phosphoric Acid Mobile» Phases. Journal of Analytical Methods in Chemistry, 2015, vol. 1, pp. 6. DOI: 10.1155/2015/732918

3. Castañeda-Ovando A., Pacheco-Hernández L., Paez E., Rodríguez J.A., Galan-Vidal C.A. Chemical studies of anthocyanins: A review. Food Chemistry, 2009, vol. 113, pp. 859-871. DOI: 10.1016/j.foodchem.2008.09.001

4. Casteele K.V. [et al.]. Separation of some anthocyanidins, anthocyanins, proanthocyanidins and related subtances by reversed phase high performance liquid chromatography. Journal of chromatography, 1983, vol. 259, pp. 291-300.

5. Hertog M.G., Hollman P.C., Katan M.B., Kromhout D. Intake of potentially anticarcinogenic flavonoids and their determinants in adults in The Netherlands. Nutr. Cancer, 1993, no. 20, pp. 21-29. DOI: $10.1080 / 01635589309514267$ 
6. Catherine A.R., Nicholas J.M., George P. Structure-antioxidants activity relationships of flavonoids and phenolic acids. Free Radical Biology \& Medicine, 1996, no. 20, pp. 933-956. DOI: 10.1016/0891-5849(95)02227-9

7. Lee J., Wrolstad R.E., Durst R. AOAC Official Method 2005.02 Total Monomeric Anthocyanin Pigment Content of Fruit Juices, Beverages, Natural Colorants, and Wines pH Differential Method. Official Methods of Analysis of AOAC International, 2005, chapter 37, pp. 37-39.

8. Cesa S., Carradori S., Bellagamba G., Locatelli M., Antonietta Casadei M., Masci A., Paolicelli P. Evaluation of processing effects on anthocyanin content and colour modifications of blueberry (Vaccinium spp.) extracts: Comparison between HPLC-DAD and CIELAB analyses. Food Chemistry, 2017, no. 232, pp. 114-123. DOI: 10.1016/j.foodchem.2017.03.153

9. Fibigr J., Satínsky D., Solich P. A UHPLC method for the rapid separation and quantification of anthocyanins in acai berry and dry blueberry extracts. Journal of Pharmaceutical and Biomedical Analysis, 2017, no. 143, pp. 204-213. DOI: 10.1016/j.jpba.2017.05.045

10. Canuto G.A.B., Oliveira D.R., Da Conceição L.S.M., Farah J.P.S., M.F.M. Tavare. Development and validation of a liquid chromatography method for anthocyanins in strawberry (Fragaria spp.) and complementary studies on stability, kinetics and antioxidant power. Food Chemistry, 2016, no. 192, pp. 566-574. DOI: 10.1016/j.foodchem.2015.06.095

11. Li D., Li B., Ma Y., Sun X., Lin Y., Meng X. Polyphenols, anthocyanins, and flavonoids contents and the antioxidant capacity of various cultivars of highbush and half-high blueberries. Journal of Food Composition and Analysis, 2017, no. 62, pp. 84-93. DOI: 10.1016/j.jfca.2017.03.006

12. Trikas E.D., Melidou M., Papi R.M., Zachariadis G.A., Kyriakidis D.A. Extraction, separation and identification of anthocyanins from red wine by-product and their biological activities. Journal of functional food , 2016, no. 25, pp. 548-558.

13. Huang Zh., Wang B., Williams P., Pace R.D. Identification of anthocyanins in muscadine grapes with HPLC-ESI-MS. Food Science and Technology, 2009, no. 42, pp. 819-824. DOI: 10.1016/j.lwt.2008.11.005

14. Ruiz A., Hermosín-Gutiérrez I., Vergara C., Von Baer D., Zapata M., Hitschfeld A., Obando L., Mardones C. Anthocyanin profiles in south Patagonian wild berries by HPLC-DAD-ESI-MS/MS. Food Research International, 2013, no. 51, pp. 706-713. DOI: 10.1016/j.foodres.2013.01.043

15. Bridle P., Garcia-Viguera C., Tomas-Barberan F.A. Analysis of Anthocyanins by Capillary Zone Electrophoresis. Journal of Liquid Chromatography \& Related Technologies, 1996, vol. 19, no. 4, pp. 537-545. DOI: 10.1080/10826079608005518

16. Caboni M.F., Comandini P., Giampaolo B., Cardinali A., Cerretani L. CZE separation of strawberry anthocyanins with axitic buffer and comparison with HPLC. Journal of separatrion of science, 2008, no. 31, pp. 3257-3264. DOI: 10.1002/jssc.200800199

17. Shim Y., Kim S., Seo D., Park H., Ha J. Rapid method for determination of anthocyanin glucosides and free delphinidin in grapes using u-HPLC. Journal of Chromatographic Science, 2014, no. 52, pp. 629-635. DOI: 10.1093/chromsci/bmt091

18. Gao L., Mazza G. Rapid Method for Complete Chemical Characterization of Simple and Acylated Anthocyanins by High-Performance Liquid Chromatography and Capillary Gas-Liquid Chromatography. Journal of Agricultural and Food Chemistry, no. 42, pp. 118-125. DOI: 10.1021/jf00037a020

19. Liu X., Mu T., Sun H., Zhang M., Chen J. Optimisation of aqueous two-phase extraction of anthocyanins from purple sweet potatoes by response surface methodology. Food Chemistry, 2013, no. 141, pp. 3034-3041. DOI: 10.1016/j.foodchem.2013.05.119

20. Nyman N.A., Kumpulainen J.T. Determination of anthocyanidins in berries and red wine by high-performance liquid chromatography. Journal of Agricultural and Food Chemistry, 2001, no. 49, pp. 4183-4187. DOI: 10.1021/jf010572i

21. Deividas B., Ivanauskas L., Dirse V. Study of diversity of anthocyanin composition in bilberry (Vaccinium myrtillus L.) fruits. Meditsina (Kaunas, Lithuania), 2007, vol. 43 no. 12, pp. 971-977. 
22. Welch C., Wu Q., Simon J. Recent Advances in Anthocyanin Analysis and Characterization. Current Analytical Chemistry, 2008, vol. 4, no. 2, pp. 75-101. DOI: 10.2174/157341108784587795

23. Zhang Zh., Kou X., Fugal K. Comparison of HPLC Methods for Determination of Anthocyanins and Anthocyanidins in Bilberry Extracts. Journal of Agricultural and Food Chemistry, 2004, no. 52, pp. 688-691. DOI: 10.1021/jf034596w

Vu Thi Trang, Le Hoang Duc, Nguyen Hoai Thu, Le Thi Hong Hao, Nguyen Xuan Trung. Multiseparation of anthocyanins and anthocyanidins by high performance liquid chromatography combined with response surface methodology. Health Risk Analysis, 2019, no. 3, pp. 118-127. DOI: 10.21668/health.risk/2019.3.14.eng

Received: 27.05.2019

Accepted: 26.07.2019

Published: 30.09.2019 